\title{
Cómo valorar e interpretar un artículo sobre pronóstico*
}

\author{
Drs. CARLOS MANTEROLA D. ${ }^{1,2,3}$, CARMEN SANTANDER A., ${ }^{2,4}$, TAMARA OTZEN H.,
}

1 Departamento de Cirugía y Traumatología, Universidad de La Frontera. Temuco.

2 Programa de Magíster en Ciencias Médicas, Universidad de La Frontera. Temuco.

3 Programa de Doctorado en Ciencias Médicas, Universidad de La Frontera. Temuco.

${ }^{4}$ Departamento de Cirugía, Universidad de Concepción. Concepción.

5 Universidad Autónoma de Chile. Temuco.

Chile.

\begin{abstract}
How to assess and interpret a prognostic study

Prognosis is the prediction of the evolution of a given disease or event of interest and the frequency of occurrence of specific outcomes. The ideal study design to assess prognosis are cohort studies. However many prognostic studies have case-control or case series designs. Prognostic studies should include all participants with the event of interest of a determinate geographical region, to avoid bias. The measurement precision and exactitude of intervening variables and outcomes, the identification of confounding variables and an adequate follow up period, have a great influence on the methodological quality of prognostic studies. Prognosis is usually expressed as survival, disease free or remission free rates. This article provides basic methodological concepts that should be considered when evaluating a prognostic study.
\end{abstract}

Key words: Prognostic, cohort studies, rates.

\section{Resumen}

Pronóstico es una predicción de la evolución de una enfermedad o evento de interés (EI), que se asocia a posibles resultados del EI y a la frecuencia con la cual se puede esperar que se produzcan. El diseño ideal para estudiar pronóstico son los estudios de cohortes, no obstante lo cual, se ha verificado que un importante número de artículos sobre pronóstico corresponden a estudios de casos y controles, y series de casos. Los estudios sobre pronóstico se deben realizar con una población integrada por todas las personas con el EI de una región geográfica definida; es decir, deben basarse en una descripción completa de los individuos estudiados para asegurar una muestra reducida de sesgos. La calidad metodológica de un estudio sobre pronóstico es influenciada por la precisión y exactitud con la que se definen y miden las variables de exposición y resultado; así como por la prolijidad con la que se identifican las potenciales variables de confusión. Involucran un

*Recibido el 20 de agosto de 2012 y aceptado para publicación el 25 de septiembre de 2012.

Los autores no refieren conflictos de interés.

Parcialmente financiado por proyecto DI09-0060 de la Dirección de Investigación Universidad de La Frontera.

Correspondencia: Dr. Carlos Manterola D.

Casilla 54-D, Temuco, Chile. Fax: 56-45-325761

cmantero@ufro.cl 
período de seguimiento de los sujetos en estudio suficiente para que se produzcan los resultados de interés relevantes. Para describir pronóstico se utilizan tasas; y las que se utilizan con mayor frecuencia son las de supervivencia a 5 años, letalidad, mortalidad específica por enfermedad; las tasas de respuesta, remisión y recidiva. El objetivo de este artículo es entregar conceptos metodológicos básicos que se han de considerar cuando se valora e interpreta un estudio sobre pronóstico.

Palabras clave: Pronóstico, estudio de cohortes, estudios longitudinales, estudios de seguimiento, sesgos, riesgo, análisis de supervivencia, Kaplan-Meier.

\section{Introducción}

El pronóstico corresponde a una predicción de la evolución de una enfermedad o evento de interés (EI) después de su inicio ${ }^{1}$. El término, hace referencia a posibles resultados de una enfermedad o EI y a la frecuencia con la que se puede esperar que se produzcan ${ }^{2}$.

En los estudios sobre pronóstico se agrupan pacientes que tienen un EI en común; se realiza el seguimiento del o los grupos a partir de un momento determinado y se miden ulteriormente los resultados. Se identifican además condiciones asociadas a un resultado concreto, las que se denominan factores asociados (FA), factores pronósticos (FP) y factores de riesgo $(\mathrm{FR})^{1}$.

La capacidad para poder establecer un pronóstico es de importancia fundamental en la toma de decisiones. Por una parte, ayuda a la entrega de información adecuada al paciente y familiares para decidir respecto de alternativas diagnóstico terapéuticas; y por otra, a la adecuada planificación y administración de recursos a nivel de salud pública.

El objetivo de este artículo es entregar conceptos metodológicos básicos que se han de considerar cuando se valora e interpreta un estudio sobre pronóstico.

\section{Tipos de diseño y su nivel de evidencia}

Considerando el planteamiento fundamental de un escenario de pronóstico de una enfermedad o EI ¿cuál es el pronóstico de...? o ¿cuáles son los FP o los FR de...? se puede suponer que el responderlas involucra una serie de variables a considerar entre los que el seguimiento hasta la medición del EI es de gran relevancia.

Cualquier artículo debería declarar de forma explícita el diseño utilizado en la conducción del estudio, y los artículos referentes a pronóstico no son una excepción.

El principal tipo de diseño para estudiar pronóstico son las revisiones sistemáticas de estudios de cohortes y los estudios de cohortes individuales. Estos últimos corresponden a un diseño de tipo observacional, longitudinal y analítico; que puede tener un carácter prospectivo o retrospectivo; y en el que se seleccionan un grupo o grupos de sujetos en base a la presencia o ausencia de un FR para un EI. Al inicio del estudio, todos los individuos deben encontrarse libres del EI en estudio y son observados por un período de tiempo para evaluar la ocurrencia del EI en estudio ${ }^{3,5}$. Las ventajas de este tipo de estudios son: que el investigador elige o precisa los grupos en estudio y evalúa características definidas, realizando controles en el tiempo para evaluar la aparición del resultado de interés (estrategia poderosa para valorar incidencia e investigar asociación causal ${ }^{2,5}$; que permite al investigador medir variables importantes en forma completa y precisa disminuyendo sesgos al obtener la información de forma retrospectiva. Entre las desventajas se encuentran la de ser estudios costosos debido a los recursos necesarios para seguir a un gran número de sujetos a lo largo del tiempo. Son ineficientes en la investigación de EI o resultados infrecuentes, ya que se debe incluir un número de individuos mucho mayor de los que presentan el EI. Además, no se dispone de resultados por un largo tiempo 2,5 .

Otros diseños de que permiten estudiar de forma adecuada el pronóstico, los FR y los FP son los ensayos clínicos ${ }^{3-6} ; \mathrm{y}$, se ha verificado además, que un importante número de artículos sobre pronóstico corresponden a estudios de casos y controles, y series de casos prospectivas y retrospectivas ${ }^{7-10}$.

En los escenarios de pronóstico, suele existir asociado a ellos los términos asociación y causalidad; o relación Causa y Efecto; de tal forma que el objetivo principal de un estudio sobre pronóstico es determinar causalidad o asociación, en la relación agente etiológico-EI. Si en un estudio sobre pronóstico, se determina que el azar, el sesgo y la confusión son improbables para la explicación del cambio en el curso de un EI, se puede concluir que hay asociación estadística; lo que no necesariamente representa una relación causa-efecto, pues una asociación estadísticamente significativa no implica indefectiblemente causalidad.

Hay criterios que pueden ayudar en los juicios de causalidad, incluyendo la fuerza de asociación, la credibilidad biológica de la hipótesis, la consistencia de los hallazgos así como otra información relacionada con la secuencia temporal ${ }^{11-13}$. El razonamiento básico para establecer una relación causal 
es la secuencia de eventos, es decir, que la causa esté presente antes que se produzca el efecto. Pero antes de establecer que dos o más factores tienen relación causa-efecto es necesario demostrar que la asociación entre ellos es real o válida; donde el efecto del azar (error aleatorio) y los sesgos (errores sistemáticos), es mínimo ${ }^{14}$. Estos últimos son de especial relevancia, pues pueden ocurrir en cualquier fase del proceso de evaluación de una asociación y provocan una comparación distorsionada dentro del modelo de razonamiento causa-efecto ${ }^{15,16}$.

En resumen, los efectos del azar y el sesgo en la evaluación de una asociación están relacionados con la calidad metodológica del estudio.

Por lo anteriormente expuesto, se puede señalar que el nivel de evidencia de los diseños de investigación clínica se encuentra directamente relacionado con la fuerza y tamaño de la asociación causal, en el entendido que estos nos informan de la proximidad con el valor real de la estimación. Desde este punto de vista, el mejor nivel de evidencia para estudios sobre pronóstico lo dan las RS de estudios de cohortes con o sin meta-análisis ${ }^{17}$, seguido por los estudios de cohortes individuales, los estudios de casos y controles y las series de $\operatorname{casos}^{18}$.

\section{La población}

Los estudios sobre pronóstico se realizan en forma óptima con una población integrada por todas las personas con la enfermedad o EI de una región geográfica definida $^{1,19}$. Sin embargo, la mayoría de los estudios de pronóstico se basan en muestras clínicas no directamente relacionadas con las poblaciones geográficas, razón por la cual cobra importancia la descripción de las características de los pacientes, el entorno en el que fueron identificados y la forma en la que se realizó el muestreo, de modo que el lector pueda decidir si resulta adecuado generalizar los resultados de un estudio determinado a otra población ${ }^{1,2,12}$. El número de individuos de los grupos a estudio debe ser el necesario para entregar una adecuada precisión y poder, de forma tal de dar validez interna a los resultados 2 : por ende, estos estudios requieren de una apropiada estimación del tamaño de la muestra.

\section{Definición y medición de las variables}

Las descripciones del pronóstico deben incluir todo el intervalo de manifestaciones de la enfermedad o EI que se consideren importantes para los sujetos en estudio. Los resultados importantes pueden ser resumidos en 5 conceptos: muerte, enfermedad, malestar, incapacidad e insatisfacción.
La calidad metodológica de un estudio sobre pronóstico es influenciada por la precisión y exactitud con la que se definen y miden las variables de exposición y resultado así como la prolijidad con la que se identifican los potenciales confundentes o variables de confusión. Las variables de desenlace o resultado y de exposición deben definirse en forma objetiva y explícita, especificando la forma en que serán medidas en todos los sujetos del estudio y cómo se llevará a cabo el análisis de los resultados; de tal forma que sea posible reproducir la experiencia en otra población ${ }^{1,12}$. Por otra parte, es fundamental al igual que en cualquier estudio la forma en que los resultados son medidos y expresados.

Las variables de desenlace o resultado deben ser medidas utilizando criterios estandarizados que se aplicarán a todos los sujetos del estudio por igual. Los investigadores encargados de realizar las mediciones deben estar enmascarados para evitar sesgos, ya que existe la posibilidad que si el investigador sabe que un sujeto presenta un FR de potencial importancia podría realizar evaluaciones más frecuentes o completas en búsqueda de la variable desenlace esperada (sesgo de sospecha diagnóstica). Por otro lado, en el caso de investigadores que evalúen muestras biológicas (biopsias, sangre, etc.); estos podrían ver su juicio diagnóstico influenciado al conocer las características clínicas del sujeto del cual proviene la muestra (sesgo de expectación).

\section{El seguimiento}

Los estudios de cohorte sobre pronóstico deben partir de un punto determinado en el curso de la enfermedad (tiempo cero) que se ha de precisar claramente y que corresponde al mismo para todos los individuos. Este, suele definirse en etapas tempranas en el curso de la enfermedad o EI (inicio de síntomas inequívocos o inicio de una intervención) ${ }^{1,12}$.

El seguimiento de los sujetos debe realizarse durante un período suficiente de tiempo para que se produzcan los resultados de interés relevantes en el mayor número de individuos. De lo contrario, los resultados subestimarán la realidad y estarán sesgados. Debe establecerse claramente un protocolo de seguimiento indicando la forma en que se evaluarán los pacientes en relación a los tiempos y medición de resultados ${ }^{20}$.

Al reportar las pérdidas de seguimiento debe analizarse la causa de éstas, las características de los sujetos perdidos y si existen diferencias entre los sujetos que completaron seguimiento y los que no lo completaron. Conocer esta información resulta relevante para poder identificar si existe alguna relación entre las características de los individuos que perdieron seguimiento y la variable desenlace. Por 
ejemplo, si los pacientes que no acudieron a control por encontrarse demasiado enfermos son excluidos, puede subestimarse el pronóstico de la enfermedad en estudio.

Existen estrategias para minimizar las pérdidas de seguimiento. Excluir a los sujetos que tengan planeado mudarse durante la conducción del estudio, obtener información relevante que permita conocer el estado vital y de ser posible localizar al paciente en caso de perder el seguimiento ${ }^{2,20}$.

\section{Los sesgos}

El sesgo corresponde a un error sistemático que, en cualquier etapa del proceso de investigación que ocurra, tiende a producir resultados que se desvían de los valores verdaderos. Existen un gran número de sesgos definidos, algunos característicos de cada diseño de investigación. Sin embargo, la mayoría puede incluirse en tres categorías: de selección, de medición y de confusión 12,17,20. Los sesgos de selección se producen cuando se comparan grupos de sujetos que, además de diferir en los factores principales del estudio, también difieren en otros que pueden afectar al resultado del mismo; los sesgos de medición ocurren cuando los métodos de medición difieren entre los grupos de sujetos en estudio; y los sesgos de confusión, cuando dos factores se encuentran asociados y el efecto de uno se confunde o queda distorsionado por el efecto del otro.

Por otra parte, en los estudios de cohortes sobre pronóstico, el sesgo puede crear diferencias aparentes entre los grupos en estudio, cuando en realidad éstas no existen; o enmascarar diferencias cuando realmente existen.

Existen sesgos particulares para este tipo de estudios; como el de muestreo (sujetos en estudio son diferentes de aquellos a los que se generalizan los resultados), y el de migración (sujetos de una cohorte abandonan su grupo inicial porque dejan el estudio o se trasladan a otro grupo); ambos correspondientes a sesgos de selección. El efecto de estos se puede minimizar o controlar a través de diversas estrategias que pueden aplicarse durante el diseño, conducción y análisis de la investigación; entre las que destacan la asignación aleatoria, la restricción, el emparejamiento, la estratificación, la estandarización y el ajuste multivariable.

\section{Aplicación de herramientas estadísticas y medidas de asociación}

Para describir pronóstico y resumir el curso de una enfermedad o EI se utilizan tasas, que expresan en forma sucinta la información. Las tasas más frecuentemente utilizadas son las de: supervivencia a
5 años, letalidad, mortalidad específica, respuesta, remisión y recidiva.

Además de aplicar estadística descriptiva, los estudios de pronóstico permiten obtener medidas de asociación con el fin de comparar pronósticos en sujetos con características diferentes, las que constituyen los posibles FP. Los efectos de estos posibles FP relacionados entre sí pueden expresarse como una razón de riesgos o razón de riesgo instantáneos (similar al riesgo relativo), que consideran el tiempo transcurrido hasta el acontecimiento. Al utilizar varias variables combinadas se pueden generar reglas de predicción que permiten estimar probabilidades de un resultado determinado en base a un conjunto de características de los sujetos en estudio.

Los tipos de pruebas estadísticas a utilizar para establecer asociación entre dos variables dependen del tipo de variables a comparar. Para variables dicotómicas tenemos el test de $\chi^{2}$ (Mantel-Haenszel y Pearson), el test exacto de Fisher y la regresión logística. Para variables de tipo ordinal tenemos el test de Mantel-Haenszel extendido y el modelo de regresión logística ordinal. Para variable dependiente continua y variable independiente dicotómica T-test; $y$ en casos de variable independiente nominal u ordinal, análisis de varianza o ANOVA.

Al interpretar pronósticos es mejor conocer la probabilidad media de que los pacientes con una afección determinada presenten un resultado en cualquier punto de tiempo, para lo que se recurre a los análisis de supervivencia (SV); los que permiten estimar y representar cualquier resultado de interés: muerte, recidiva o recurrencia, curación, ausencia de síntomas o inactividad de una enfermedad.

La forma más directa de estudiar SV sería conformar una cohorte de sujetos con el EI que se encuentren en un mismo punto del curso de éste y someterlos a observación hasta que todos presenten el resultado de interés, graficando dicha información en una curva; lo que resulta impracticable. Para ello, existen los denominados análisis de SV, cuyo método habitual se conoce como curvas de KaplanMeier; y que pueden aplicarse a cualquier variable dicotómica que se produzca una sola vez durante el seguimiento (por ejemplo muerte, recurrencia, etc.). Estas curvas, representan de forma gráfica el análisis de SV (Figura 1); de tal forma que en el eje de ordenadas se muestra la probabilidad de SV estimada y en el eje de abscisas el período de tiempo transcurrido desde el inicio de la observación. De este modo, la probabilidad de sobrevivir en un punto del tiempo se calcula a partir de la probabilidad acumulada de sobrevivir en cada uno de los intervalos de tiempo que lo precedieron (Tabla 1). Ahora bien, si en el curso del seguimiento se pierden sujetos por razones distintas al resultado de interés, estos se 
Tabla 1. Método para calcular curva de SV de Kaplan-Meier

\begin{tabular}{|c|c|c|c|c|}
\hline $\begin{array}{c}\text { Columna } 1 \\
\text { Tiempo SV (meses) }\end{array}$ & $\begin{array}{l}\text { Columna } 2 \\
\text { n de orden }\end{array}$ & $\begin{array}{c}\text { Columna } 3 \\
\text { Orden de observaciones } \\
\text { no censuradas (r) }\end{array}$ & $\begin{array}{c}\text { Columna } 4 \\
(n-r) /(n-r+1)\end{array}$ & Columna 5 \\
\hline $3+$ & 1 & -- & -- & -- \\
\hline 6 & 2 & 2 & $18 / 19=0,95$ & 0,95 \\
\hline $7+$ & 3 & -- & -- & -- \\
\hline $7+$ & 4 & -- & -- & -- \\
\hline 8 & 5 & 5 & $15 / 16=0,94$ & 0,89 \\
\hline 10 & 6 & 6 & $14 / 15=0,93$ & 0,83 \\
\hline 11 & 7 & 7 & $13 / 14=0,93$ & 0,77 \\
\hline $11+$ & 8 & -- & -- & -- \\
\hline $11+$ & 9 & -- & -- & -- \\
\hline 12 & 10 & 10 & $10 / 11=0,91$ & 0,70 \\
\hline 13 & 11 & 11 & $9 / 10=0,90$ & 0,63 \\
\hline 13 & 12 & 12 & $8 / 9=0,89$ & $0,56^{*}$ \\
\hline $14+$ & 13 & -- & -- & -- \\
\hline $16+$ & 14 & -- & -- & -- \\
\hline $20+$ & 15 & -- & -- & -- \\
\hline $20+$ & 16 & -- & -- & -- \\
\hline 22 & 17 & 17 & $3 / 4=0,75$ & 0,42 \\
\hline 32 & 18 & 18 & $2 / 3=0,67$ & 0,28 \\
\hline 34 & 19 & 19 & $1 / 2=0,50$ & 0,14 \\
\hline 36 & 20 & 20 & 0 & 0,0 \\
\hline
\end{tabular}

*Cuando hay un tiempo de SV (13 meses) con valores de SV diferentes, se utiliza como estimador el valor más bajo $(0,56)$.

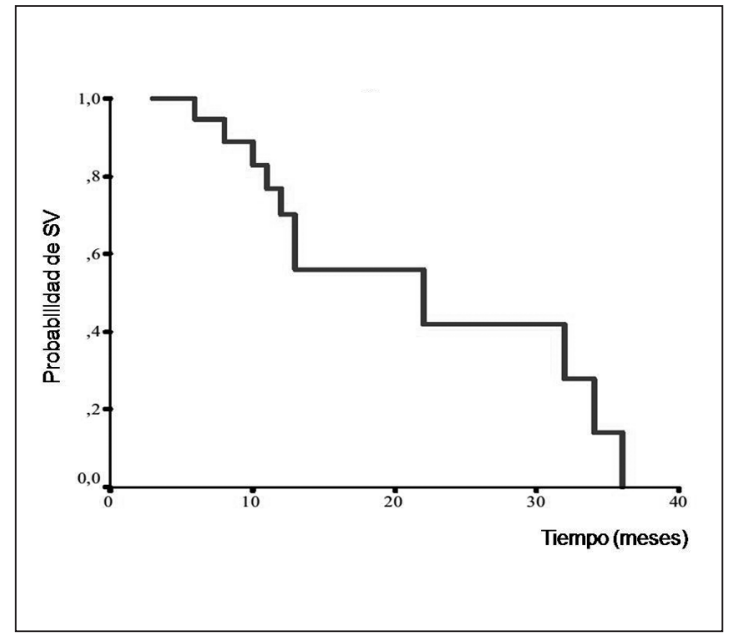

Figura 1. Curva de Kaplan-Meier graficada con los valores de la Tabla 1, en que se aprecia la probabilidad de SV en el tiempo, medida en meses. consideran "censurados" y dejan de contabilizarse en el denominador (Figura 1).

De este modo, el eje de las ordenadas representa la probabilidad estimada de SV de los integrantes de una cohorte hipotética y no el porcentaje de supervivientes de una cohorte real; entendiendo que los puntos de una curva representan la mejor estimación de un conjunto de datos, de la probabilidad de SV de los integrantes de una cohorte, dependiendo del número de sujetos en estudio (precisión de la estimación). Por lo que la precisión a la izquierda de la curva tiende a ser mayor que a la derecha de la curva cuando existe un mayor número de individuos en riesgo.

\section{Sistemas de puntuación y listas de comprobación}

A continuación se mencionan iniciativas de distintos grupos de estudio de metodología de la inves- 
tigación, que han aportado diferentes herramientas que ayudan en la valoración general y específica de la calidad metodológica de los artículos de pronóstico.

STROBE: "Strengthening the Reporting of Observational Studies in Epidemiology". Fue desarrollado para guiar a los autores a mejorar la calidad de la publicación de estudios observacionales, entre los que destacan cohortes, casos y controles. Esta declaración, consta de 5 dominios (Título y resumen, introducción, métodos, resultados y discusión), que incluyen 22 ítems; 18 de los cuales son de aplicación general para estudios de cohortes, de casos y controles, y de corte transversal; y 4 que son específicos para cada uno de los tres diseños antes mencionados. También requiere por parte de los autores de la generación de un diagrama de flujo ${ }^{21}$.

MINCIRPr: "Metodología de Investigación en Cirugía". Fue desarrollado para valorar la calidad metodológica de estudios de distintos diseños en relación a artículos de pronóstico. Está compuesto por 25 ítems agrupados en 6 dominios: el primero, relacionado con la variable desenlace; el segundo con la variable de exposición; el tercero, con el seguimiento; el cuarto, con el análisis y las conclusiones; el quinto, con el tipo de diseño y las variables de confusión; y el sexto, con el tamaño de la muestra. De esta forma, se genera una puntuación final que puede fluctuar entre 25 y 125 puntos, asignando 25 puntos al estudio de menor calidad metodológica y 125 puntos al de mejor calidad metodológica ${ }^{22}$. MINCIRPr.: "Metodología de Investigación en Cirugía". Segunda versión de la iniciativa anterior, desarrollada para valorar la calidad metodológica de estudios relacionados con pronóstico. Está compuesto por 8 ítems agrupados en 4 dominios; el primero relacionado con el diseño del estudio, el segundo con el tamaño de la población estudia$\mathrm{da}$, el tercero con la metodología empleada y el cuarto con el análisis y las conclusiones. De esta forma, se genera una puntuación final que puede fluctuar entre 7 y 60 puntos, con un punto de corte de 33 puntos que dicotomiza el constructo "calidad metodológica"; constituyéndose de este modo en una herramienta más simple que la anteriormente descrita ${ }^{23}$.

En resumen quien escribe un manuscrito relacionado con pronóstico, o quien lee un artículo de este tipo debe exigir al menos un objetivo claro, preciso y conciso respecto del escenario de la investigación que se realizó; la mención explícita del diseño empleado con los respectivos detalles metodológicos inherentes a este; y la mención y ejecución de herramientas estadísticas y medidas de asociación, o al menos los números necesarios para poder calcular estos valores.

\section{Aplicación de herramientas de lectura de artículos de pronóstico}

Existen criterios para la evaluación e interpretación de estudios de pronóstico. Estos son los siguientes:

1. Definición de una pregunta de Investigación. Un artículo de pronóstico debe intentar responder una pregunta de investigación bien formulada. En su defecto, se requiere de la definición de objetivos; los que se han de enunciar de forma clara y precisa.

2. Población de estudio. Se ha de especificar la forma de selección y de muestreo de la población a estudio, de forma de asegurar a una muestra representativa de la población a la que se desea extrapolar resultados. Del mismo modo, se han de establecer de forma clara los criterios de inclusión y exclusión.

3. Tamaño de la muestra. Se debe estimar el tamaño de la muestra.

4. Definición de variables. Se han de definir las variables de exposición, de desenlace o resultado y de confusión (si se logran identificar). Así mismo, se deben establecer el método y unidades de medida; y que estos sean iguales para todos los sujetos en estudio. Finalmente, asegurarse que el método de medición sea válido y confiable.

5. Análisis del seguimiento. Cerciorarse que el seguimiento es lo suficientemente prolongado y exhaustivo y que se enuncia el porcentaje de pérdida de seguimiento (descripción de los sujetos perdidos y las causas de pérdida del seguimiento).

6. Análisis de resultados y conclusiones. Determinar medidas de riesgo y asociación; y utilización de modelos de asociación o predictivos. Verificar que las conclusiones del estudio son coherentes con la metodología empleada.

7. Validez externa. Comprobar que los resultados del estudio en evaluación pueden ser aplicados a la población de interés del lector.

\section{Ejemplo}

A modo de ejemplo se analizará el artículo de Ogino et al, publicado en $2009^{24}$, según la escala MinCirPr.

\section{Dominio 1 (variable de desenlace)}

¿Se define la variable de desenlace? Sí. SV a 5 años en pacientes con cáncer de colon.

¿El método para medir la variable de desenlace es válido y confiable? Sí. Los datos respecto de fallecimientos y sus causas fueron obtenidos de registros médicos en quienes completaron seguimiento 
y a través de entrevistas a familiares, revisión de registros médicos y nacionales de estadísticas vitales en aquellos que perdieron seguimiento.

¿Se define la unidad de medida de la variable de desenlace? Sí. SV global a 5 años por cáncer de colon.

¿Una adecuada proporción de la muestra tiene datos completos de la variable de desenlace? Sí. $98 \%$ tiene datos completos de estado vital y causa de muerte.

¿El método y las unidades de medición de la variable de desenlace son los mismos para todos los pacientes? Sí. En todos los casos se obtiene SV global y grafica curva de SV.

\section{Dominio 2 (variable de exposición)}

¿Se define la variable de exposición? Sí. Expresión positiva de la ciclina $\mathrm{D}_{1}$ en muestras de tejido tumoral de pacientes con cáncer de colon.

¿El método para medir la variable de exposición es válido y confiable? Sí. Técnicas de inmunohistoquímica con anticuerpos monoclonales (160 muestras al azar por 2 observadores independientes obteniendo correlación positiva de 0,83 ).

¿Se define las unidades de medida de la variable de exposición? No. Se clasifica la expresión de ciclina $\mathrm{D}_{1}$ en: sin expresión, expresión débil y expresión moderada/intensa; pero no se especifica en términos cuantitativos cómo se define esta clasificación.

¿Una adecuada proporción de la muestra tiene datos completos de la variable de exposición? Sí. Se analizaron muestras del 100\% de los pacientes incluidos en el estudio.

¿El método y las unidades de medición de la/las variables de exposición son los mismos para todos los pacientes? Sí. Se describe el método para análisis de expresión de ciclina $\mathrm{D}_{1}$, que fue aplicado a todas las muestras.

\section{Dominio 3 (seguimiento)}

¿Se enuncia el porcentaje de pérdida de seguimiento? No.

¿Se describen las características de los sujetos perdidos? No.

¿Se hicieron esfuerzos por recolectar la información de los sujetos perdidos? Sí. Se recurrió a diferentes estrategias: contactar a familiares que aportaran dicha información, revisión de registros médicos o búsqueda en registros nacionales.

¿Las causas de pérdida de seguimiento son descritas? No. Sólo se menciona que algunos casos no asistieron a controles ni pudieron ser ubicados, no especificando su número ni causas.

¿Se analizaron las diferencias entre las características de los sujetos con pérdida de seguimiento y los que no? No.

\section{Dominio 4 (análisis y conclusiones)}

¿Con los datos presentados es posible determinar las medidas de riesgo y asociación? Sí. Se entregan tablas indicando número total de datos positivos para cada variable considerada como las medidas de riesgo correspondientes.

¿Se realizan modelos de asociación o predictivos? Sí. Se utiliza modelo de riesgos proporcionales de Cox para calcular razones de riesgo de muerte según estado de expresión de ciclina $\mathrm{D}_{1}$ tumoral ajustado por edad, sexo, índice de masa corporal, historia familiar de cáncer colorrectal, ubicación y estadio tumoral; y estado de las variables de confusión relacionadas con eventos moleculares. El análisis de SV se realizó según Kaplan-Meier.

¿El modelo elegido es adecuado según el diseño del estudio? Sí. Con excepción de la edad, año de diagnóstico y metilación LINE-1 (variables continuas), todas las demás variables son categóricas.

¿Las conclusiones del estudio son coherentes con el objetivo y la metodología empleadas? Sí. Tras el análisis multivariado con ajuste se encontró que la expresión positiva de ciclina $\mathrm{D}_{1}$ en tejido tumoral se asocia tanto a una menor mortalidad específica por cáncer de colon como mortalidad general en los pacientes en estudio.

\section{Dominio 5 (diseño y variables de confusión)}

¿Se enuncia claramente el diseño del estudio? Sí. Corresponde a un estudio de cohorte.

¿Considera variables de confusión? Sí. Considera eventos moleculares expresados en el tejido tumoral tales como la inestabilidad microsatelital, mutación de BRAF y KRAS, hipometilación LINE1, COX-2, p53, p21 y p27, entre otros, los que se han visto asociados al pronóstico de pacientes con cáncer de colon en otros estudios.

¿Se definen adecuadamente las variables de confusión? Sí. Se enumeran y se describe cómo serán evaluadas.

¿Los métodos para medir las variables de confusión son válidos y confiables? Sí. Para todas ellas se utilizan métodos de inmunohistoquímica de alta sensibilidad y especificidad, tales como reacción de polimerasa en cadena.

\section{Dominio 6 (tamaño de la muestra)}

¿Hay cálculo del tamaño de la muestra? No. Sólo se indica que son 602 pacientes cuyas muestras de tejido pudieron ser obtenidas y analizadas.

Se justifica el tamaño de la muestra? No.

Puntaje total de la escala (dominios $1+2+3+$ $4+5+6): 21+25+9+20+20+2=97$ puntos. Se trata entonces de un estudio de calidad metodológica intermedia. 


\section{Referencias}

1. Fletcher R. Epidemiología Clínica. Lippincott Williams Wilkins. $4^{\circ}$ Edición 2007. Capítulo 7: Pronóstico. Capítulo 8: Tratamiento.

2. Hulley S. Designing Clinical Research: An Epidemiologic Approach. Lippincott Williams \& Wilkins. $2^{\circ}$ Edition 2001.

3. Manterola C, Zavando D, Grupo MINCIR. Cómo interpretar los "Niveles de Evidencia" en los diferentes escenarios clínicos. Rev Chil Cir. 2009;61:582-95.

4. Manterola C, Muñoz S, Bustos L. Estrategias de investigación. Diseños observacionales. $3^{\mathrm{a}}$ parte. Estudio de cohortes. Rev Chil Cir. 2001;53:410-4.

5. Manterola C, Bustos L. Estrategias de investigación. Diseños experimentales. Ensayo clínico. Rev Chil Cir. 2001;53:498-503.

6. Manterola C. Estudios observacionales. Los diseños utilizados con mayor frecuencia en investigación clínica. Rev Med CLC 2009;20:539-48.

7. Manterola C, Pineda V, Vial M. Acerca de los ensayos clínicos en el ámbito quirúrgico. Reflexiones, dificultades y desafíos. Rev Chil Cir. 2009;61:482-9.

8. Pineda V, Manterola C, Vial M, Losada H. ¿Cuál es la calidad metodológica de los artículos referentes a terapia publicados en la Revista Chilena de Cirugía? Rev Chil Cir 2005;57:500-7.

9. Manterola C, Pineda V, Vial M, Losada H; MINCIR Group. What is the methodologic quality of human therapy studies in ISI surgical publications? Ann Surg. 2006;244:827-32.

10. Manterola C, Busquets J, Pascual M, Grande L. What is the methodological quality of articles on therapeutic procedures published in Cirugía Española? Cir Esp. 2006;79:95-100.

11. Manterola C, Grande L. Methodological quality of articles on therapeutic procedures published in Cirugía Española. Evaluation of the period 2005-2008. Cir Esp. 2010;87:244-50.

12. Hennekens $\mathrm{CH}$, Buring JE. Epidemiology in Medicine. Boston: Little Brown and Company; $1^{\circ}$ Edition 1989.

13. Kelsey JL, Thompson WD, Evans AS. Methods in
Observational Epidemiology. New York: Oxford University Press; 1986.

14. Feinstein AR. An outline of cause-effect evaluations En: Clinical epidemiology the architecture of clinical research. W.B. Saunders Company;1985. pp. 39-52.

15. Manterola C, Pineda V, Grupo MINCIR. El valor de "p" y la "significación estadística". Aspectos generales y su valor en la práctica clínica. Rev Chil Cir. 2008;60:86-9.

16. Castellanos-Olivares A, Vásquez-Márquez I, WacherRodarte N. ¿Qué es un sesgo? En: Ramiro HM, SaitaKamino O, editores. Temas de medicina interna, epidemiología clínica. México. Interamericana-McGrawHill;1994. pp. 237-47.

17. Fletcher RH, Fletcher SW, Wagner EH. Epidemiología clínica. Aspectos fundamentales. $2^{\mathrm{a}} \mathrm{ed}$. México: Masson Wlliams (Wilkins, España, SA;1998. pp. 7-10.

18. Manterola C. Revisión sistemática de la literatura síntesis de la evidencia. Rev Med CLC 2009;20:897-903.

19. Díaz F. introducción a los estudios de cohorte en epidemiología y al análisis de supervivencia. Med UNAB 2005;8:43-53.

20. Sackett D. Clinical Epidemiology: A Basic Science for Clinical Medicine. Little, Brown and Company. $2^{\circ}$ Edition 1991.

21. Vandenbroucke JP, von Elm E, Altman DG, Gøtzsche PC, Mulrow CD, Pocock SJ, et al; STROBE initiative. Strengthening the Reporting of Observational Studies in Epidemiology (STROBE): explanation and elaboration. Ann Intern Med. 2007;147:W163-94.

22. Losada H, Manterola C, Pineda V, Vial M, Sanhueza A. Grupo MINCIR. Diseño de una escala para la evaluación de calidad metodológica de estudios de pronóstico. Rev Chil Cir 2009;61:59-72.

23. Zavando D, Manterola C. Grupo MINCIR. Validación inicial de una escala de medición de calidad metodológica para artículos de pronóstico. Rev Med Chile (en prensa).

24. Ogino S, Nosho K, Irahara N, Kure S, Shima K, Baba Y, et al. A Cohort Study of Cyclin D1 Expression and Prognosis in 602 Colon Cancer Cases. Clin Cancer Res. 2009; 15:4431-8. 